\section{Locomotion like a wheel?}

SIR - Since the time of Sumer, our conception of efficient movement has been shaped by the wheel. Vehicles using wheels are efficient because they result in smooth rides. Wheel-like rides are possible even when legs are used for locomotion. Engineers, realizing the advantages of legged locomotion, have built many-legged robots that also minimize repeated accelerations and decelerations. Despite the efficiency of wheel-like motion, animals do not have wheels, possibly because the formation of a wheel-like structure is biologically problematic ${ }^{1}$ and wheels are not necessarily advantageous on all surfaces ${ }^{2,3}$.

The quest for efficient, wheel-like dynamics in legged robots led us to ask whether any animal in an appropriate environment actively moves like a wheel using its legs or body. Hoop snakes that catch their tail in their mouth to roll better may be mythical ${ }^{4}$, but desert spiders can pull in their legs and roll passively down a slope to escape danger ${ }^{5}$. Some coelenterates can cartwheel or roll by alternate attachment of the pedal disk and tentacles ${ }^{6}$. From a handful of candidates such as these, we thought that the most likely species to use active, wheel-like motion was a crustacean reported to roll along the beach ${ }^{7}$.

The small stomatopod Nannosquilla decemspinosa is found on the sand beaches of the Pacific coast of Panama. We
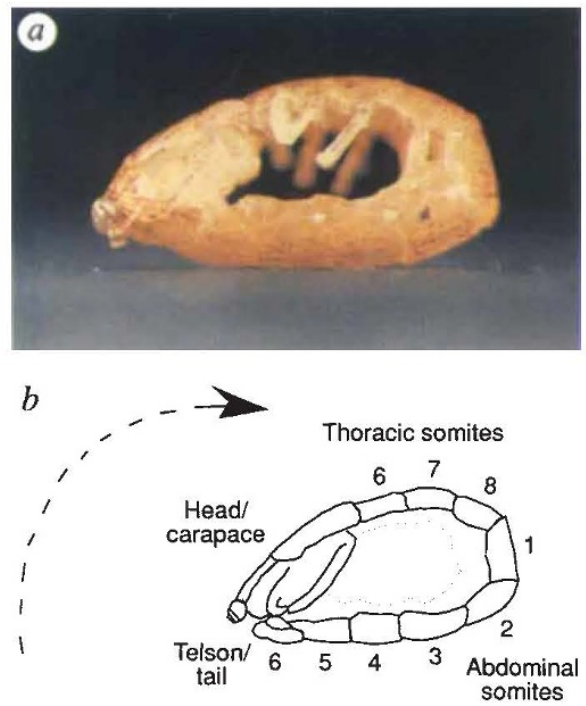

C

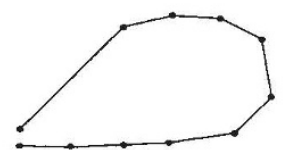

FIG. 1 a, Backwards somersault of a stomatopod. $b$, The body of $N$. decemspinosa consists of a head/shell, thorax, abdomen and tail. c, Spatial model of a stomatopod used for motion study. videotaped the somersaulting of this shrimp-like crustacean to see if it moved like a true wheel (Fig. 1). Because of their elongated body and short, laterally projecting legs, adults cannot walk when out of the water. When washed from their burrows and cast up on the beach by waves, they move across the sand by

in synchrony that also function as a single, effective 'leg'8. Animals seldom use wheel-like locomotion even when it is possible, and instead rely on pendulum or spring-like mechanisms that require repeated oscillations of their bodies. Because most legged robots are dynamically more similar to turtles than faster and more manoeuvrable animals, perhaps future robotic designs can benefit from the transfer of biologically inspired ideas

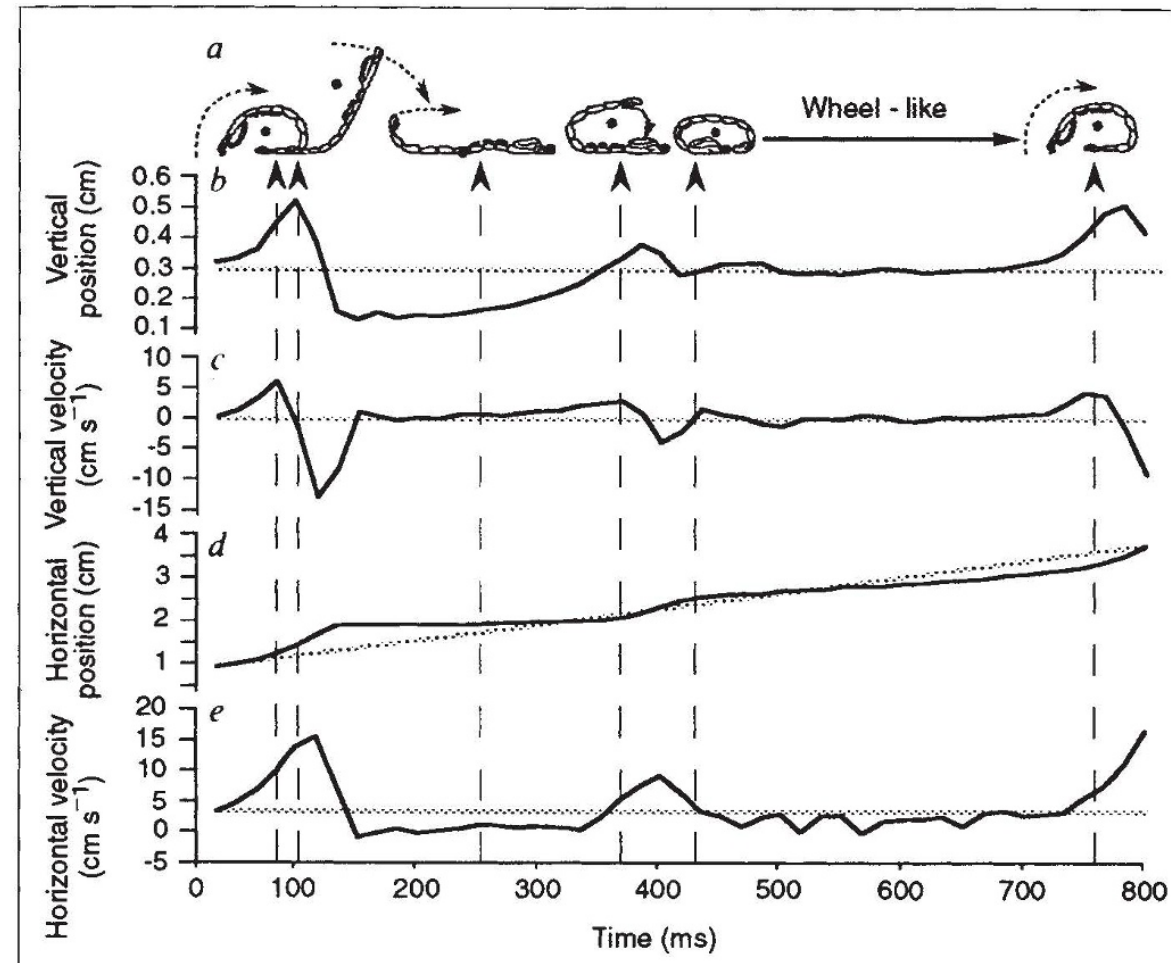

FIG. 2 Motion of the stomatopod's centre of mass (filled circle) during one backwards somersault. $a$, The stomatopod uses its tail as a single leg to lift its body up and move it forwards. After a slight pause on its back, the stomatopod lifts its tail and curls it up over the body. The body then acts as a leg to push the tail section up and over. When the tail touches the ground the stomatopod rolls like a true wheel with the centre of mass at the hub. $b$, vertical position; $c$, vertical velocity; $d$, horizontal position; $e$, horizontal velocity.

backwards somersaults for as far as $2 \mathrm{~m}$, completing as many as 20-40 consecutive rolls ${ }^{7}$. Stomatopods actively propelled themselves forwards at 72 revolutions per min or 1.5 body lengths per $\mathrm{s}(3.5 \mathrm{~cm}$ per $\mathrm{s})$ and rolled as a true wheel for 40 per cent of each revolution (Fig. 2). Sixty per cent of a cycle was marked by large accelerations and decelerations similar to the patterns generated by legged walkers and runners. When the animal could not capitalize on wheel-like motion, its body functioned as a single leg to thrust itself upwards and forwards.

The unique stomatopod somersaulter accelerated and decelerated its body each cycle by using a single, effective 'leg', as do many-legged animals who also had been presumed to move like wheels (supposedly, the more legs operating in a wave, the more wheel-like is the movement). Instead, insects, crabs and centipedes tip or bounce by using several legs that represent alternatives to wheel-like motion ${ }^{8}$. Who knows how many of these alternatives are waiting to be discovered in nature?

\section{Robert Full}

Kathleen Earls

Mary Wong

Roy Caldwell

Department of Integrative Biology,

University of California,

Berkeley,

California 94720, USA

1. Gould, S. J. Nat. Hist. 90, 42-48(1981).

2. LaBarbara, M Am Nat 121,395-408 (1983).

3. Walker, 1. Acta Biotheor. 39, 151-155 (1991).

3. Walker, 1. Acta Biotheor. 39, 151-155 (1991).
4. Higgins, W. W. Anthology of Hoopsnake Tales (Onio Valley Folk Research Project, Ross County Historical Society. 1959)

5. Henschel, J. R. S. Afr. J. Sci. 86, 151-152 (1990).

6. Trembley, A. Memoires Pour Servir L L'histoire d'un Genre de Polypes d'eu Dounce, a Bras en Forme de Connes 323 (Verbeek, Leide, 1744)

7. Caidwell, R. L. Nature 282, 71-73(1979).

8. Full, R. J. in Biological Neural Networks in invertebrate Neuroethology and Robots (eds Beer, R., Ritzmann, R. \& McKenna, T.) 3 (Academic, New York, 1993). 